\title{
Philosophiques
}

\section{Kant rencontre Aristote là où la raison rencontre l'appétit}

\section{Peter Railton}

Volume 28, numéro 1, printemps 2001

La nature des normes

URI : https://id.erudit.org/iderudit/004972ar

DOI : https://doi.org/10.7202/004972ar

Aller au sommaire du numéro

\section{Éditeur(s)}

Société de philosophie du Québec

\section{ISSN}

0316-2923 (imprimé)

1492-1391 (numérique)

Découvrir la revue

\section{Citer cet article}

Railton, P. (2001). Kant rencontre Aristote là où la raison rencontre l'appétit. Philosophiques, 28(1), 47-67. https://doi.org/10.7202/004972ar

\section{Résumé de l'article}

Nous pouvons tous, je crois, reconnaître la justesse de la thèse d'Aristote à l'effet que le véritable raisonnement pratique a pour résultat non pas une simple croyance à propos du caractère désirable, ou même du caractère obligatoire, d'un acte, mais plutôt l'initiation effective d'une action. Cette thèse donne lieu à une énigme : comment la délibération, archétypiquement une inférence propositionnelle rationnelle ( logon ), peut-elle logiquement aboutir à un acte ? L'action présuppose la motivation, mais la motivation est une force appétitive active, et non pas une proposition concernant le désir ou le caractère désirable d'un acte (elle est donc alogon ). La raison pratique n'est pas similaire aux substances qui peuvent manifestement être mélangées les unes aux autres, mais elle est censée constituer une fusion normative régie par des principes - le choix est un appétit délibéré , comme le dit Aristote. Comment ceci est-il possible ? Les premiers pas vers une réponse peuvent être trouvés à un endroit surprenant : chez Kant. Dans la Métaphysique des Moeurs, Kant présente une théorie du désir comme appétit pour un objet par le biais d'une idée non-sensorielle de cet objet, et ce, par opposition à une inclination, un appétit direct basé sur la sensation. Kant emploie cette notion du désir pour expliquer à la fois la volonté et la possibilité d'une action accomplie par devoir : la volonté appartient à la faculté de désirer, et le devoir est l'action motivée par un désir pour un acte par le biais d'une idée appropriée de cet acte. Kant nomme notre susceptibilité à ce type de désir laquelle constitue une condition nécessaire à la moralité le sentiment moral . Ce sentiment rend compte du caractère pratique de la délibération morale et de son rapport au plaisir et à la douleur pratiques d'une façon qui aurait, je crois, plu à Aristote. Le devoir est donc un exemple d'un appétit délibératif. Ce schème kantien n'est pas simplement intéressant d'un point de vue historique : il s'accorde remarquablement bien avec certains travaux récents en psychologie motivationnelle et hédonique. 


\title{
Kant rencontre Aristote là où la raison rencontre l'appétit
}

\author{
PETER RAILTON \\ University of Michigan \\ prailton@umich.edu
}

RÉSUMÉ. - Nous pouvons tous, je crois, reconnaître la justesse de la thèse d'Aristote à l'effet que le véritable raisonnement pratique a pour résultat non pas une simple croyance à propos du caractère désirable, ou même du caractère obligatoire, d'un acte, mais plutôt l'initiation effective d'une action. Cette thèse donne lieu à une énigme : comment la délibération, archétypiquement une inférence propositionnelle rationnelle (logon), peut-elle logiquement aboutir à un acte ? L'action présuppose la motivation, mais la motivation est une force appétitive active, et non pas une proposition concernant le désir ou le caractère désirable d'un acte (elle est donc alogon). La raison pratique n'est pas similaire aux substances qui peuvent manifestement être mélangées les unes aux autres, mais elle est censée constituer une fusion normative régie par des principes le choix est un appétit délibéré, comme le dit Aristote. Comment ceci est-il possible ? Les premiers pas vers une réponse peuvent être trouvés à un endroit surprenant : chez Kant. Dans la Métaphysique des Mœurs, Kant présente une théorie du désir comme appétit pour un objet par le biais d'une idée non-sensorielle de cet objet, et ce, par opposition à une inclination, un appétit direct basé sur la sensation. Kant emploie cette notion du désir pour expliquer à la fois la volonté et la possibilité d'une action accomplie par devoir : la volonté appartient à la faculté de désirer, et le devoir est l'action motivée par un désir pour un acte par le biais d'une idée appropriée de cet acte. Kant nomme notre susceptibilité à ce type de désir laquelle constitue une condition nécessaire à la moralité le sentiment moral. Ce sentiment rend compte du caractère pratique de la délibération morale et de son rapport au plaisir et à la douleur pratiques d'une façon qui aurait, je crois, plu à Aristote. Le devoir est donc un exemple d'un appétit délibératif. Ce schème kantien n'est pas simplement intéressant d'un point de vue historique : il s'accorde remarquablement bien avec certains travaux récents en psychologie motivationnelle et hédonique.

ABSTRACT. - We all can, I think, appreciate the justness of Aristotle's view that genuine practical reasoning ends not in a mere belief about the desirability, or even obligatoriness, of an act, but in the actual initiation of action. This yields a puzzle: how can deliberation, archetypically a rational, propositional inference (logon), logically terminate in an act? Action presupposes motivation, yet motivation is an active appetitive force, not a proposition about desire or desirability (hence alogon). Unlike substances can obviously be mixed together, but practical reason is supposed to a principled, normative fusing - choice is deliberated appetite, as Aristotle puts it. How is this possible? The beginnings of an answer can be found in a surprising place: Kant. In The Metaphysics of Morals Kant gives a theory of desire as appetite for an object through a non-sensory idea of it, in contrast to inclination, a sensation-based direct appetite. Kant uses this notion of desire to explain both will and the possibility of action from duty: will belongs to the faculty of desire, and duty is action motivated by a desire for an act

PHILOSOPHIQUES 28/1 — Printemps 2001, p. 47-67 
through an appropriate idea. Kant terms our susceptibility to this sort of desire moral feeling, a necessary condition for morality. It accounts for the practicality of moral deliberation, and its linkage to practical pleasure and pain, in a way Aristotle would, I think, have found congenial. Duty, then, is an example of deliberated appetite. The Kantian scheme is not merely of historical interest: it fits surprisingly well with recent work in motivational and hedonic psychology.

\section{Introduction : L'énigme d'Aristote.}

Les personnes rationnelles sont censées être guidées par quelque chose qui mérite le nom de "raison pratique ». Dans ses moments les plus explicites, celle-ci prend la forme d'une délibération pratique. Selon la théorie classique développée par Aristote, la délibération pratique, contrairement au raisonnement théorique, n'a pas pour conclusion une croyance ou un jugement, mais plutôt une action, ou du moins une tentative d'action : "son terme ultime constitue le point de départ de l'action ${ }^{1}$.

Nous sommes habitués à l'idée d'un raisonnement avec des prémisses et des conclusions propositionnelles; mais qu'est-ce que ce serait que de construire un raisonnement dont la conclusion s'apparenterait à une action ? Il aurait certainement des prémisses quelque peu différentes de celles des inférences déductives que l'on retrouve dans les textes de logique. Après tout, c'est une caractéristique plutôt générale du raisonnement valide qu'il n'y a rien dans la conclusion qui ne soit pas d'une certaine façon déjà contenu dans les prémisses. Si l'action, ou la tentative d'action, implique une fusion de la pensée et de la motivation afin de générer un mouvement dirigé, alors la pensée et la motivation doivent d'une façon ou d'autre être toutes deux présentes parmi les prémisses de la délibération pratique. Ceci semble effectivement être le point de vue d'Aristote :

Il est par conséquent tout à fait logique que les deux principes qui apparaissent moteurs soient l'appétit et la réflexion exécutive. Car c'est l'objet de l'appétit qui déclenche le mouvement. Et la raison pourquoi la réflexion est motrice, c'est qu'elle a pour point de départ cet objet de l'appétit. ... Mais voilà : l'intelligence, de son côté, ne déclenche manifestement pas de mouvement sans appétit, puisque le souhait (boulesis) est un appétit et que, lorsque le mouvement se règle sur le raisonnement, il se règle aussi selon le souhait (boulesis); tandis que l'appétit, de son côté, déclenche un mouvement qui déroge au raisonnement; puisque le désir et une espèce d'appétit. ${ }^{2}$

1. Aristote, De L'âme, trad. franç. par R. Bodéus, Paris, Flammarion, 1993, $433 a 16$.

2. Ibid., 433a17-26 ; Bodéus traduit ici boulesis par "souhait ", mais comme le terme grec s'applique surtout aux conclusions d'une réflexion pratique ou d'une résolution législative, le terme "volonté » semble préférable (ou de conserver le terme grec) plutôt que de suggérer qu'il s'agit d'une espérance telle qu'un souhait sans force active. (On verra aussi une ressemblance importante avec la conception de la volonté chez Kant.) Bodéus traduit orexis un terme tout à fait générique pour désigner la motivation, mais lié en particulier avec les désirs appétitifs, comme le faim — par « appétit ». 
Et dans l'Éthique à Nicomaque: « ... le principe de l'action (humaine), c'est la décision ; (or) la décision, c'est une intelligence appétitive ou un appétit intellectif. »[1139a31-b5 $]^{3}$

L'appétit (orexis), nous dit Aristote, n'est pas cognitif — il appartient à la partie de l'esprit qui est alogon (parfois traduit «non rationnelle » ou « irrationnelle ») ; le raisonnement et l'intellect, par contraste, appartiennent à cette partie de l'esprit qui est logon (parfois traduit « rationnelle ») $)^{4}$. Mais l' « appétit intellectif » ou l'« intelligence appétitive » sembleraient alors similaires à de l'huile et de l'eau. Comment peuvent-ils s'intégrer par le biais d'une délibération pratique en une action- et ce, conformément à une règle ? L'huile et l'eau peuvent se mélanger si on les agite, mais ce type d'émulsion est tout à fait contraire à une combinaison qui soit « réglementée » d'un point de vue chimique - aucune des substances ne modifie réellement l'autre, et avec le temps les deux éléments vont simplement se reséparer. Cependant, le choix est une fusion, " un désir délibératif des choses qui dépendent de nous $»^{5}$ et "la décision est chose commune à la pensée et à l'appétit " ${ }^{6}$.

Aristote dresse ici pour nous plusieurs énigmes, deux desquelles feront l'objet principal de cet article. Premièrement, si la délibération pratique doit constituer une forme valide de raisonnement, elle devra contenir parmi les éléments qui constituent effectivement ses "prémisses " un élément de désir - non pas simplement une pensée quant à ce qui « est désirable " (ou bon), mais un véritable désir, un état appétitif de l'organisme qui soit capable de produire un movement. Puisque l'appétit est alogon, il ne peut être le produit du raisonnement comme tel, et ainsi, d'après Aristote, la délibération pratique ne produit pas en elle-même des fins étant donné que le désir est présupposé et amené à jouer un rôle au sein du contexte délibératif, plutôt que d'être créé par lui ${ }^{7}$. Mais si tel est le cas, comment l'intellect peut-il donner à l'appétit ou au désir une application définie et réglementée, ou une forme pratique au sein de l'action ? Ceci est une énigme tout à fait générale concernant l'action, mais même si nous avions sa solution en main, une seconde énigme subsisterait. Comment l'action qui est régie par des normes, comme l'action morale est souvent jugée devoir l'être - i.e. régie par des principes qui sont catégoriques ou indépendants de tout penchant préalable - est-elle possible ? Certes, le sens commun nous mène à mettre en opposition l'action accomplie par devoir et l'action accomplie par désir. Mais, si Aristote a raison de dire que le raisonnement pratique implique toujours un élément de désir, comment le raisonnement moral pourrait-il jamais être pratique, ou

3. Ibid., p. 244n. Ailleurs dans ce texte, Aristote, Éthique à Nicomaque, trad. franç. par J. Tricot, Paris, Vrin, 1983. Les références aux lignes de l'original sont approximatives.

4. Cf. Aristote, Éthique à Nicomaque, trad. franç. par J. Tricot, Paris, Vrin, 1983, 1102a28.

5. Ibid, 1113a10

6. Aristote, Mouvement des animaux, trad. franç. par R. Bodéus, cité en De l'âme, p. 245n.

7. Aristote, Éthique à Nicomaque, $1112 \mathrm{~b}$ 


\section{0 · Philosophiques / Printemps 2001}

encore, comment l'action rationnelle pourrait-elle jamais être accomplie par devoir ?

Les premiers pas vers une solution aux deux énigmes peuvent, je crois, être trouvés dans l'œuvre d'un philosophe souvent considéré comme étant le défenseur par excellence de l'opposition entre le devoir et le désir - Kant.

\section{La solution de Kant}

Je crains que nous ne ferons aucun progrès en cette matière avant d'avoir précisé d'avantage la nature du désir. L'œuvre de Kant peut sembler un étrange endroit où aller chercher une théorie du désir, mais pourtant dans son livre qui mit tant de temps à paraître, la Métaphysique des Mours (dans une section intitulée "Du rapport des facultés de l'esprit humain aux lois morales »), Kant offre un modèle convaincant du désir et de sa relation à l'action régie par des normes. L'on s'attendrait à ce que Kant désigne la volonté comme étant la source d'une force de motivation qui soit assez suffisante pour générer une action et même capable d'opérer en dépit d'un penchant préalable. Et c'est en effet ce qu'il fait. Pourtant, loin d'opposer la volonté au désir, Kant situe la volonté à un endroit tout à fait aristotélicien, soit dans la "faculté de désirer » : "La faculté de désirer ... s'appelle la faculté de faire ou de ne pas faire, à sa convenance [nach Belieben]. ... La faculté de désirer, dont le principe interne de détermination, même la convenance par conséquent, se trouve dans la raison du sujet, s'appelle volonté.$^{8}$ "

Si avoir de la force de volonté c'est avoir une volonté puissante, et si la volonté est située au sein de la faculté de désirer, alors manifester de la force de volonté est une question d'avoir (entre autres choses) une faculté de désirer puissante. Mais comment une faculté de désirer (Begierde), même puissante, pourrait-elle être, par nature, différente du " penchant » (Neigun) ? Et que signifierait le fait que ce qui « convient » à une faculté de désirer git « au sein de la raison du sujet " plutôt que « dans l'objet » du désir en tant que tel,

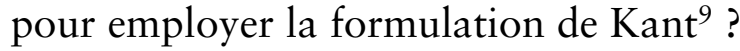

Nous devons commencer par dire un mot sur la structure du désir, et par conséquent sur la "faculté de désirer ». Le désir, nous dit Kant, est toujours lié au plaisir et au déplaisir. Il écrit :

8. Kant, Emmanuel, Métaphysique des Mœurs, trad. franç. par Joëlle Masson et Olivier Masson, dans Oeuvres philosophiques, tome III, Alquié, Ferdinand, dir., Bibliothèque de la Pléiade, Paris, Gallimard, 1986, vi, 213. Les autres citations de Kant qui paraissent dans le texte sont tirées des Fondements de la Métaphysique des Mours, trad. franç. par V. Delbos, dans Oeuvres philosophiques, tome II, Alquié, Ferdinand, dir., Bibliothèque de la Pléiade, Paris, Gallimard, 1984. Les numéros de pages sont ceux de l'édition de l'Académie de l'œuvre de Kant.

9. Kant, Métaphysiques des Mours, vi, 213. 
Avec le désir ou l'aversion est premièrement toujours lié le plaisir ou le déplaisir, dont la réceptivité s'appelle sentiment; mais l'inverse n'est pas toujours vrai. Il peut en effet exister un plaisir qui ne soit pas lié à un désir de l'objet mais seulement à la représentation que l'on se fait d'un objet (savoir si l'objet existe ou non restant indifférent). ${ }^{10}$

Le fait de rêvasser agréablement à ce qu'on sait ne pas être possible n'est pas un " plaisir pratique ${ }^{11}$ ni un désir véritable, comme le reconnaîtrait Aristote. Ainsi, il ne donne pas lieu à un choix et à une action ; il s'agit d'un " plaisir purement contemplatif ou satisfaction inactive ", et c'est pour cette raison que la rêverie ne dépend d'aucune pensée à l'effet que l'objet contemplé existe réellement ou est accessible ${ }^{12}$. Par contraste, lorsqu'il fait face à l'inexistence ou à l'inacessibilité, le désir occasionne de la frustration ou de la déception, qui sont tous deux des formes de "déplaisir pratique ».

Bien que le plaisir et le déplaisir soient toujours associés au désir, ils ne sont pas toujours à l'origine du désir :»Deuxièmement, le plaisir ou le déplaisir pris à un objet du désir ne précède pas toujours non plus le désir et n'en saurait toujours être regardé comme la cause, mais il peut aussi être considéré comme son effet. " ${ }^{13}$.

Comment ceci fonctionne-t-il ? Ce ne sont pas tous les désirs qui se fixent sur leurs objets en vertu du plaisir qu'ils offrent ou promettent. Mais du moment que le désir s'est fixé sur un objet, il se manifeste (entre autres choses) par un degré de satisfaction lorsqu'on se voit progresser vers l'atteinte de l'objet, ou un degré d'insatisfaction si l'on voit que notre progression est entravée ou qu'elle échoue. Le plaisir et le déplaisir sont, en euxmêmes, des états intrinsèquement motivants, fondamentaux et à peine explicables - des états que nous partageons probablement avec plusieurs espèces. Ils ont, par ailleurs, des rôles fonctionnels caractéristiques, en connection avec l'appétit, par exemple., "... le plaisir et le déplaisir ne peuvent pas être expliqués clairement en eux-mêmes ; l'on peut tout au plus préciser quelles conséquences ils ont dans certaines circonstances, de manière à les rendre reconnaissables dans la pratique. " ${ }^{14}$.

Si on a faim, alors manger (ou le fait de se mouvoir avec succès vers l'acte de manger) plaira, et le plaisir nous motivera à continuer jusqu'à ce que notre faim ait diminuée ou soit disparue. (Kant nous rappelle, dans un contexte différent, l'adage qu'à bon appétit il ne faut point de sauce). Par contraste, si nous n'avons de prime abord pas faim, alors manger peut, en soi, être une corvée, et même être désagréable — on doit se gaver lorsque la maladie nous coupe l'appétit et qu'on veut respecter l'ordonnance du docteur de

10. Ibid., vi, 211.

11. Ibid., vi, 212.

12. Ibid., vi, 212.

13. Ibid., vi, 211.

14. Ibid., vi, 212. 


\section{2 · Philosophiques / Printemps 2001}

prendre du poids, ou lorsqu'on n'a pas faim mais qu'on désire faire plaisir à un hôte trop anxieux. Toutefois, même dans ces cas, un type de plaisir peut être occasionné par le fait de réussir à manger. Peut-être pas un plaisir associé à la nourriture ou à l'acte de manger en tant que tels, mais le plaisir de parvenir à un but que nous nous sommes fixés, en dépit de la résistance du corps. Par contraste, si nous ne parvenons pas à manger dans de tels cas, nous ressentirons du déplaisir et de la frustration, même si notre corps est soulagé. Nous savons que quelqu'un a un véritable désir d'accomplir avec succès une tâche ou de réussir une activité (et non pas seulement le simple souhait d'une plus grande réussite en général) par la force et le caractère de sa frustration envers - et par sa motivation à surmonter — les obstacles à sa réalisation. Le désir nous pousse donc à être la cause de ce que nous avons en tête, et non pas seulement un appréciateur de l'idée de ce que nous avons en tête : "La faculté de désirer est la faculté d'être, par ses représentations, cause des objets de ces représentations $»{ }^{15}$.

Bien qu'il implique l'appétit, le désir n'est pas un simple appétit. L'appétit qui est associé à la privation et qui est excité par la sensation est un état que nous partageons avec plusieurs espèces. Mais l'appétit qui est associé à une idée, motivé et opérant par l'entremise de "nos représentations non-sensorielles »- est un peu plus particulier. Rappelons la remarque d'Aristote à l'effet que, grâce au désir, ce qui autrement nous paraîtrait surprenant se produit : "la réflexion est motrice » ${ }^{16}$, c'est-à-dire la "raison pratique » est possible.

Une certaine élucidation de la formule de Kant - le désir entendu comme étant le fait d'être, par ses représentations, cause des objets de ces représentations - semble indiquée. Considérez pour commencer un simple appétit comme la faim, tel que le vit un animal simple, disons un poisson. Supposons que nous avons un poisson dans un aquarium, et que nous le nourrissons en saupoudrant de la nourriture dans l'eau. Il y a un certain temps que le poisson a été nourri, et il a décidément faim ; il nage rapidement vers la nourriture et mange vigoureusement. Dans ce cas, on peut dire que la nage du poisson est " guidée par la sensation de l'objet de l'appétit », c'està-dire qu'elle est attribuable à son état de faim en conjonction avec sa détection sensorielle de la présence de nourriture. (Voir Fig. 1.)

Nous ne disons pas que le poisson est "guidé par la délibération pratique » ou qu'il est " rationnel du point de vue pratique ", même s'il parvient à utiliser son cerveau de façon efficace pour satisfaire son appétit à la lumière d'une juste perception des circonstances. Est-ce parce que le poisson ne construit aucun syllogisme pratique ? - Mais nous construirons rarement nous-mêmes de tels syllogismes, même lorsque nous agissons d'une

15. Ibid., vi, 211.

16. Aristote, De L'âme, 433a19. 
FIGURE 1

\section{Être guidé directement par la sensation et l'appétit}

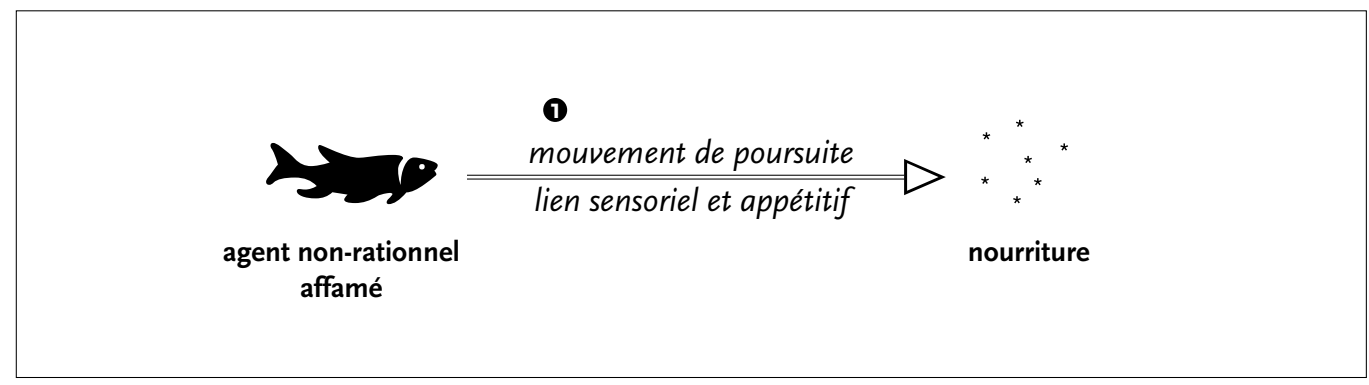

La quête est guidée par la sensation et l'appétit. Le poisson affamé suit son instinct, qui le guide vers la nourriture. Si une autre source de nourriture aussi aisément identifiable se présentait en 1, alors le poisson modifierait sa trajectoire et se dirigerait vers 1.

façon que nous qualifierions ordinairement de rationnelle d'un point de vue pratique. Le poisson est dans un état qui le porte à être incité au mouvement dirigé par la sensation de la nourriture. Son comportement est intelligent et est guidé par son cerveau, mais pour l'expliquer nous n'avons pas besoin d'invoquer l'habileté du poisson à être guidé par une idée - une représentation non-sensorielle — de la nourriture.

Considérez le comportement d'une personne qui vient tout juste d'arriver à l'hôtel dans une nouvelle ville, et qui se trouve toute seule dans sa chambre avec ses valises, vers vingt heures. Elle voit qu'il est à peu près l'heure du souper et elle a en effet très faim. Elle est fatiguée et doit travailler, mais elle sait que manger au restaurant de l'hôtel va tout simplement la déprimer elle aimerait manger un repas convenable. Elle s'est fait dire par un ami qu'on sert de la très bonne cuisine vietnamienne dans cette ville, mais que la plupart des restaurants s'affichant comme "vietnamiens " produisent en fait une variante inauthentique de la cuisine chinoise destinée à plaire aux touristes. Elle n'est pas certaine d'avoir déjà goûté à de la cuisine vietnamienne authentique, mais elle a un vif désir d'en essayer. Nous la trouvons pourtant assise sur le coin du lit scrutant un épais livre jaune. Qu'est-ce qui explique ce comportement ? "Elle a faim, elle est prête à manger . " Mais, certainement, la lecture du livre jaune n'est pas « l'objet de son appétit » — pourquoi n'avance-t-elle pas vers la nourriture comme le fait le poisson affamé ? «Et bien, elle s'avance vers la nourriture, mais d'une manière quelque peu indirecte. Son ami a mentionné le nom d'un restaurant typiquement vietnamien situé dans cette ville mais elle ne peut retrouver le bout de papier sur lequel elle l'a écrit, et elle sonde donc la liste des restaurants dans les Pages jaunes dans l'espoir qu'un nom lui sautera aux yeux. »

En effet, elle agit pour " être la cause de l'objet de son appétit », mais elle le fait en agissant de manière à " être la cause de l'objet de sa représentation » d'un type particulier de souper. Elle ne le fait certainement 


\section{Philosophiques / Printemps 2001}

pas grâce à une quelconque sensation directe de l'objet — il n'y a pas la moindre sensation de mets vietnamiens dans sa chambre d'hôtel. Mais il y a de l'information concernant la disponibilité de mets vietnamiens dans sa chambre, et elle s'est tournée vers celle-ci afin de poursuivre sa quête. L'objet de sa poursuite n'est pas une substance qui soit simplement nutritive, ni un repas qui a bon goût mais n'est pas typiquement vietnamien. Elle serait frustrée et fâchée envers elle-même si elle ne parvenait qu'à trouver un repas à emporter de cuisine américaine standard, ou si elle s'assoyait à un restaurant s'affichant comme vietnamien pour alors réaliser (trop tard!) qu'il appartient définitivement à la catégorie touristique - même si elle y trouve la cuisine assez plaisante et satisfaisante. Parce qu'elle agit sur la base d'une idée assez définie du type de repas qu'elle désire, elle peut être guidée dans son cheminement vers un objet de désir qu'elle ne voit pas ni ne connaît par rien de plus que de l'information, en opérant par inférence et délibération.

Le désir, comme le langage, tend vers un référent par l'entremise d'une image, d'un sens, ou d'une intension. (Voir Fig. 2.) Aristote écrit : « ...[les] bêtes ... possèdent bien la sensation mais n'ont pas l'action en partage. Et ce que l'affirmation et la négation sont dans la pensée, la recherche et l'aversion le sont dans l'ordre du désir ; ...le choix est un désir [orexis] délibératif... ${ }^{17}$. $\mathrm{Au}$ fur et à mesure qu'elle précise sa ligne de conduite, notre cliente affamée façonne volontairement l'opération de son appétit. Elle fait appel à ses connaissances et à son expérience antérieure ("Je devrai faire attention — les meilleurs endroits ne sont parfois pas dans les Pages jaunes » ou «Quelque soit son nom, ce n'est certainement pas le restaurant à l'énorme message publicitaire disant 'Informez-vous à propos de nos spéciaux sur les pichets de Marguarita'»), elle tient compte de ses contraintes ("Je ne veux pas prendre un taxi, ni dépenser une fortune " ou "Ce devra être un endroit où je ne me sentirai pas mal à l'aise de manger seule »), et elle a recours à certaines stratégies ( "Je ne suis pas certaine, mais ce nom me semble familier et ce restaurant se trouve dans un quartier où sont situés un certain nombre de restaurants vietnamiens. J'irai donc sur place y jeter un coup d'œil »). Elle déploie une faculté bien développée d'être, "par ses représentations, cause des objets de ces représentations " — les activités liées à sa quête d'un souper manifestent non seulement de l'appétit (pour de la nourriture), mais également le désir d'un repas (de l'appétit pour un objet par l'entremise d'une image de celui-ci). En effet, elle doit dans une certaine mesure contrôler sa faim afin d'avoir la patience, la concentration, et l'énergie requises pour mener à terme sa délibération et sa quête.

Les références aux idées, à la signification, à la délibération, au choix, et au langage ne devraient pas toutefois complètement dominer notre raisonnement concernant le rôle du désir dans l'action. Car bien qu'il soit caracté- 
FIGURE 2

\section{Être guidé par le désir \\ La faculté de désirer est la faculté d'être, par ses représentations, cause des objets de ces représentations.}

[Kant, MM, vi, 211]

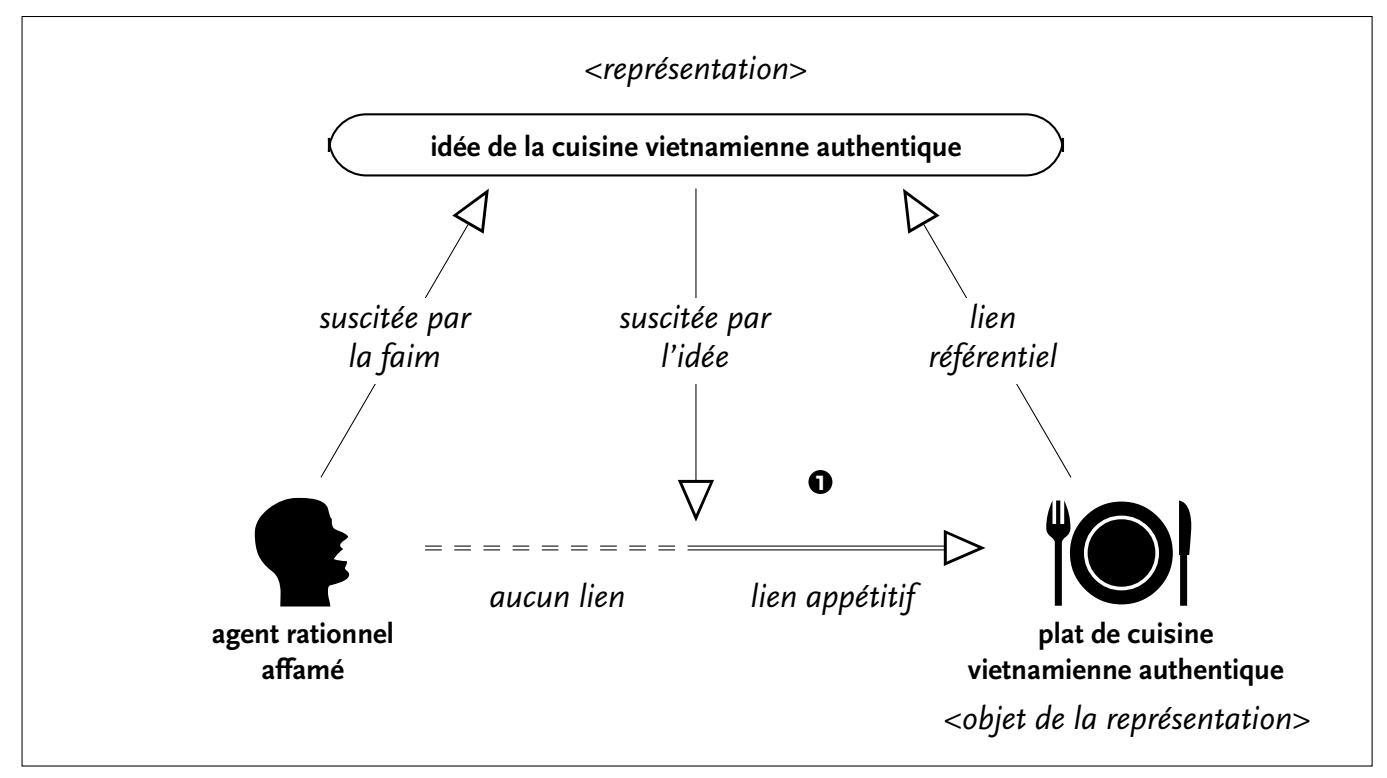

La quête est guidée par le désir ( "l'intellect appétitif »). La voyageuse affamée suit une idée qui l'oriente vers un repas. La faim la fait penser à un repas, et le souvenir de la recommandation d'un ami ramène à sa mémoire l'idée de goûter de la cuisine vietnamienne authentique. Cette idée sert de base à d'autres inférences, par exemple, celle d'aller chercher et de consulter une copie des Pages Jaunes. Si une autre source de repas aussi clairement identifiable se présentait en $1,-$ disons, un restaurant chinois attrayant qui clairement ne satisfaisait pas la description " cuisine vietnamienne authentique ", l'agent ne modifierait pas sa trajectoire et ne se dirigerait pas vers 1 . Si un livre ayant l'air sérieux intitulé Plaisirs secrets: où trouver de la cuisine vietnamienne authentique en Amérique du Nord se présentait en 1, alors l'agent altérerait sa trajectoire et se dirigerait, pour le moment, vers 1, même si ce livre n'est pas, et ne semble pas être, un repas. Le livre, contrairement au restaurant chinois, est, de façon appropriée, délibérativement lié à l'idée de "goûter de la cuisine vietnamienne authentique ». Si le livre est scellé hermétiquement, et que s'y trouve indiqué " Ne pas développer, sauf après achat ", l'agent ressentira de la frustration. Ce sentiment non-sensoriel de "déplaisir pratique » constitue une preuve de la présence d'un appétit médiatisé par une idée. Si l'agent parvient à trouver le nom d'un " restaurant vietnamien authentique » situé dans le quartier, elle sera contente lorsqu'elle y arrivera et s'assoiera, et cela, avant même de goûter la nourriture. Ce sentiment non-sensoriel de " plaisir pratique » est, lui aussi, un signe de la présence d'un appétit médiatisé par une idée.

ristique de l'action accomplie par désir qu'elle implique une certaine idée et qu'elle octroie à la délibération et au langage un rôle dans l'engagement et dans le fonctionnement de la motivation, il n'en demeure pas moins qu'il est possible d'agir par désir sans qu'une quelconque formulation linguistique explicite ou qu'une délibération active soient mises en jeu. Non seulement l'habitude et la pratique familière prédominent-elles, mais souvent les conceptions des choses impliquées dans le désir sont au plus de simples images, ou sont même si inexplicites qu'on ne peut les recouvrer qu'avec difficulté. Nous serions de très lents et piètres acteurs, sans faire mention du fait que 


\section{Philosophiques / Printemps 2001}

nous serions incapables d'initier quelque action, si toute action requérait une formulation explicite d'une pensée linguistiquement encodée et un processus délibératif conscient.

Le désir a toujours un élément cognitif, mais ce n'est que dans certains cas qu'il fait appel à quoi que ce soit qui puisse être compris comme un « jugement qu'une chose est désirable. " Tout comme il arrive souvent que nous ne prenions conscience de ce que nous croyons qu'en découvrant que quelque chose nous surprend - ou ne nous surprend pas - , il arrive fréquemment que nous ne prenions conscience de ce que nous désirons qu'en découvrant que quelque chose nous frustre - ou nous plaît. Aristote, comme Kant, suppose que toute action, indépendamment de la noblesse de l'idée à travers laquelle elle se produit, a ce lien caractéristique au «plaisir pratique " - la satisfaction associée à ce qui semble une quête fructueuse d'un objet, la frustration associée à ce qui semble être un échec dans la poursuite d'un objet. Ainsi, " tout sentiment et toute action sont toujours accompagnés de plaisir ou de douleur ${ }^{18}$, et : «[N]ous devons prendre pour signe distinctif de nos dispositions le plaisir ou la peine qui vient s'ajouter à nos actions ${ }^{19}$.

L'appétit est responsable de cette frustration ou de ce plaisir, et il a une panoplie d'autres effets. Plusieurs de ceux-ci, comme la frustration et le plaisir, sont présents même chez des animaux qui sont incapables de formuler les images ou les significations essentielles au désir. L'appétit dirige l'intérêt, fournit l'énergie pour le mouvement, affine de façon sélective la sensation, et oriente et prépare l'organisme de multiples façons. Il pourvoit la force motivationnelle qui, selon Aristote, doit servir de prémisse à toute délibération véritablement pratique. Chez les êtres capables de formuler des pensées et des inférences significatives et organisées, l'appétit oriente également cette activité de façon sélective, façonnant nos pensées et les canalisant sans permission préalable ni délibération. En effet, nous pouvons en apprendre sur ce que nous désirons en remarquant non seulement ce qui a pour nous de l'attrait (what attracts us) mais également ce qui nous distrait (what distracts us) - là où nous découvrons que notre esprit erre en dépit de notre effort de le maintenir ailleurs.

Cette référence à la distraction devrait également nous rappeler que l'influence de l'intellect sur l'appétit et l'action ne conduit pas forcément à la vérité et n'est pas toujours pour le mieux. L'appétit, en tant qu'état nonreprésentatif, ne peut être ni vrai ou faux, ni moral ou immoral. Mais le désir, de par sa nature, génère la pensée et, par conséquent, permet à la vérité ou à la fausseté, à la moralité ou à l'immoralité, d'avoir un impact sur l'activité ou de s'y manifester. Le désir, quelque soit son origine ou son objet, lie la quête d'objets à des motivateurs intrinsèques, soit le plaisir et la douleur. Il 
peut ainsi permettre à la poursuite d'une idée peu judicieuse de susciter un plaisir pratique et de la satisfaction, ou un sentiment de frustration lorsque cet effort échoue. Aristote écrit : "Nous mesurons nos actions, tous plus ou moins, au plaisir et à la peine qu'elles nous donnent. Pour cette raison encore, nous devons nécessairement centrer toute notre étude sur ces notions, car il n'est pas indifférent pour la conduite de la vie que notre réaction au plaisir et à la peine soit saine ou viciée $»^{20}$.

\section{Le penchant vs. le désir}

Or, si l'idée sur la base de laquelle l'appétit mène à l'activité dirigée est une idée de type $S$, alors nous pouvons agir de façon $S$, même si la douleur et le plaisir pratiques figurent toujours dans le processus. C'est dire que, si le plaisir et la douleur pratiques qui motivent notre activité résultent de notre désir pour $S$, il ne s'en suit pas pourtant que nous devrions être perçus comme agissant en vue d'un plaisir plutôt qu'en vue de $S$. Par conséquent, Kant remarque que le raisonnement moral peut être un raisonnement pratique en vertu de la faculté de désirer. Il ne serait pas possible d'être guidé par des normes si toute motivation était simplement stimulée par l'expérience du plaisir ou du déplaisir " pris à un objet du désir » en soi. L'expérience répétée du plaisir ou du déplaisir occasionné par un objet ou une activité donne effectivement lieu à un type d'apprentissage ou de réponse conditionnée, mais si la motivation n'avait qu'une telle source de stimulation cet apprentissage aurait les caractéristiques d'une habitude développée en vertu de la récompense et de la punition, et non pas celui d'une action accomplie par égard pour une idée ou en vue d'une fin. Kant nomme ce type de « désir habituel » le « penchant » : «... en ce qui concerne le plaisir pratique, la détermination de la faculté de désirer que ce plaisir doit nécessairement précéder comme cause s'appellera, au sens strict, désir, tandis que le désir "chronique" s'appellera penchant ${ }^{21} \ldots$

Des animaux relativement simples peuvent manifester ce type de motivation conditionnée via le plaisir et la douleur, et il est effectivement au fondement du «conditionnement opérant» des psychologues behavioristes promouvant le modèle animal. Toutefois, ces animaux sont incapables d'être guidés par l'idée d'un objet qui, même sans l'occurrence répétée d'expériences de plaisir et de douleur, serait en mesure de mettre en branle les méchanismes motivationnels de plaisir et de douleur liés à l'appétit ${ }^{22}$. Cette capacité à ressentir de l'appétit à travers des idées - et donc grâce au raisonnement

20. Ibid.,1105a3-5.

21. Kant, Métaphysiques des Moeurs, vi, 212.

22. Le conditionnement opérant lie un stimulus " conditionné » - et donc en principe conventionnel et sans ressemblance avec le stimulus "non-conditionné »(la nourriture, par exemple) - avec l'appétit. Après avoir été "conditionné ", un stimulus "non-gustatif ", comme une sonnerie ou une ampoule éclairée, peut exciter un mouvement appétitif vers la 


\section{8 · Philosophiques / Printemps 2001}

basé sur les idées - ne peut exister que chez des êtres munis d'une faculté de désirer, au sein de laquelle des " représentations » d'objets ou d'actions créés par la pensée, l'imagination ou l'inférence, peuvent être liés à l'appétit et ainsi constituer une source de plaisir ou de douleur. Ceci n'est plus un cas de penchant, mais demeure toutefois un cas de désir. Kant écrit : "lorsque le plaisir ne peut suivre une détermination antérieure de la faculté de désirer, il devra être nommé plaisir intellectuel, et l'intérêt pris à l'objet, intérêt de la raison $[. .].[\mathrm{O}] \mathrm{n}$ ne peut substituer aucun intérêt du penchant à un pur intérêt de la raison $» 23$.

Kant peut maintenant expliquer comment il est possible de résoudre notre énigme, à savoir comment la délibération pratique peut en tout temps inclure une prémisse appétitive ou désidérative en dépit du fait que ses conclusions peuvent être indépendantes de tout penchant préalable. Il peut du moins l'expliquer s'il parvient à nous convaincre de l'authenticité, d'un point de vue psychologique, de cette distinction entre le penchant et le désir, et de la possibilité que puisse figurer parmi nos désirs un appétit pour l'action par le biais d'une idée pratique normative.

\section{Un exemple}

Heureusement pour nous, Kant traite le devoir moral comme un paradigme de l'action motivée par un "intérêt de la raison " plutôt qu'un "intérêt de penchant ». Nous pouvons donc aborder les deux questions posées au principe de notre enquête à la fois. Pour ce faire, nous ne sommes pas obligés de souscrire à la doctrine kantienne selon laquelle seule l'action effectuée par devoir moral a véritablement une valeur morale. Pour le moment, notre question n'est pas celle de savoir quelle espèce de motivation est bonne, mais plutôt celle de savoir quels types de motivations sont possibles.

Si le devoir moral est le résultat de la délibération pratique ou de la raison pratique, et si tout raisonnement pratique a parmi ses prémisses un élément appétitif, alors quel est cet élément dans le cas de l'action accomplie par devoir ? Kant insiste fortement sur le fait que même un sentiment aussi généreux que la bienveillance ne peut causer l'action effectuée par devoir l'action causée par un tel sentiment relève du penchant préalable. Pourquoi en est-il ainsi ?

Prenons un exemple : l'acte d'aider un étranger dans le besoin, disons le fait d'aider un étranger confus que vous voyez par hasard dans une gare mouvementée pendant que vous vous dirigez vers votre train. L'étranger transporte une valise de planches à pâte et un calepin usé dans lequel sont

nourriture. En effet, ce lien « conventionnel » est une sorte de «prototype animal » du lien qu'on trouve au sein du désir humain entre une représentation (une idée, par exemple) et la poursuite appétitive de l'object de la représentation.

23. Kant, Métaphysique des Mours, vi, 211-212. 
inscrits un nom, un numéro de téléphone et, jugeant par les apparences, aucun mot de français. Il fait face au flux de gens qui le dépassent avec empressement, son calepin en main, pointant le nom du doigt et demandant clairement de l'aide. Vous avez vous-mêmes, imaginons-nous, quitté le bureau plus tard qu'à l'habitude, et vous devez préparer le souper pour votre famille avant de sortir pour la soirée avec un collègue qui sera (vous le savez) offusqué par votre retard, qu'il soit ou non justifié. Il semble peu probable que l'étranger et vous partagiez un langage.

Soit. Ce qui serait de loin le plus facile à faire serait de passer à côté de lui dans l'immense flux de gens rentrant du travail. Pourtant, après l'avoir dépassé, vous vous arrêtez et vous vous retournez. Avec un sentiment d'inévitabilité ( "Je devrai presque certainement prendre le train suivant»), de malchance ("Pourquoi fallait-il que je le remarque ? ), de suspicion ("Il y a des équipes de pickpockets qui s'y prennent exactement de cette façon»), de culpabilité défensive ( « Je devrai m'excuser à ma famille et à mon collègue, et ils m'en tiendront rigueur »), de colère que personne d'autre ne s'arrête ( « Ce n'est certainement pas tout le monde qui doit se presser pour rentrer à la maison afin de préparer le souper avant d'aller rencontrer un collègue susceptible - qu'en est-il de ceux qui se dirigent vers un bar ? »), et de crainte d'une humiliation ( «Le numéro sera erroné, ou encore la personne qui répondra parlera une langue que je ne comprends pas »), vous marchez vers l'étranger avec une intention marquée, vous vous efforcez d'afficher un sourire rassurant, et vous signifiez votre intention de l'aider.

Vous parvenez heureusement - bien que lentement - à le mettre en contact avec un membre de la famille qu'il doit rencontrer. Vous acceptez en vitesse ses remerciements sincères quoique distraits, vous appelez à la maison, vous vous excusez auprès de votre époux qui vous parle d'un ton glacial et auprès de vos enfants mécontents, et vous vous dirigez vers le prochain train. Pourquoi avez-vous fait ceci ? Si Aristote a raison, vous avez agi par désir. Mais ceci n'est pas quelque chose que vous vouliez vraiment faire. Quelle preuve y a-t-il qu'un désir, au sens propre du terme, motive votre action ?

Si le principal désir à l'œuvre était un penchant pour la bienveillance ou une passion de longue date (un véritable goût, que certaines personnes semblent avoir) pour l'acte de "venir à la rescousse » de personnes dans le pétrin, ceci se manifesterait de plusieurs façons. La bienveillance et la passion pour la rescousse vous disposent, par exemple, à non seulement être plus enclins à porter de l'aide - ils vous donnent également un certain goût pour celui-ci. Comme la faim, ils vous rendent plus enclins à imaginer avec désir des situations d'assistance dramatique, à être à l'affût de situations requérant de l'aide, à vous enthousiasmer face à leur possibilité éminente et à ressentir une certaine frustration si votre impulsion de prêter secours est entravée. Ceux qui ressentent une passion pour la rescousse peuvent même se sentir lésés ou déçus si, après qu'ils aient remarqué une urgence et qu'ils se soient précipités pour agir, le problème se résoud soudainement ou un autre secou- 
reur apparaît et le résoud avant eux. Ces plaisirs vécus ou envisagés surgissent en vous avant que vous ayez jugé que l'acte de porter de l'aide était requis ou même approprié du point de vue moral. En effet, la bienveillance ou le désir de porter de l'aide se manifestent de ces façons même lorsqu'on juge qu'il n'est pas moralement requis ou qu'il serait même moralement inapproprié de venir à la rescousse - admettons, par exemple, que quelqu'un mérite, et même nécessite grandement — l'effet disciplinant d'éprouver pleinement (pour une fois !) la difficulté de la situation que ses actes ont engendrée, plutôt que de s'en voir épargner comme d'habitude grâce à la bienveillance des autres.

Quelle sorte de preuve y aurait-il du fait que vous avez aidé l'étranger « par devoir » ou en vertu d'un " intérêt de la raison », plutôt qu'uniquement en vertu d'un " appétit préalable "? La plupart des sentiments qui vous ont été attribués dans la description ci-dessus étaient négatifs - un sentiment d'inévitabilité, de frustration, de crainte, de colère, une attitude défensive, et ainsi de suite. Ce sont des signes que, quels que soient les autres facteurs en jeu, vous agissez aussi bien par aversion que par désir. Cette distinction est réelle d'un point de vue psychologique — même au niveau psychologique, il existe des emplacements et des mécanismes substantiellement différents pour les émotions négatives et les émotions positives. Cette aversion démontre que vous agissez, en partie, en dépit de forts penchants préalables.

Mais si vous ne vous sentiez que de l'aversion pour le fait de revenir sur vos pas afin d'offrir de l'aide, pourquoi le feriez-vous ? Vous n'avez pas été traîné par une force externe - l'impulsion est venue de l'intérieur. Il ne semble pas non plus que vous ayiez réagi par crainte d'une punition externe pour ne pas avoir aidé, ou par attrait pour une récompense externe pour avoir aidé - ce cas n'offrait aucune promesse ni de l'une ni de l'autre. Voici un étranger aux moyens limités et vous n'êtes qu'un individu dans la foule. Évidemment, il y a toujours la possibilité que ce que vous aviez en aversion n'était que la punition interne de votre propre conscience. Il y a certainement quelque chose de juste dans cette idée qu'une conscience est à l'œuvre, mais pour que tel soit le cas, nous devons déjà présupposer un type particulier de motivation.

Quelle forme ce «type particulier de motivation » peut-il avoir — pourrait-il être un désir ? Il ne peut être un penchant préalable pour le fait d'offrir de l'aide puisque l'actualisation d'un tel penchant serait en soi moralement neutre, comme nous l'avons remarqué. Ce doit être une sorte d' " appétit pour un objet ou un acte " par le biais d'une conception spécifiquement morale de cet objet ou de cet acte, qu'il s'agisse de prêter une assistance bienveillante ou (dans des circonstances quelque peu différentes) de la refuser. S’il s'agissait véritablement d'un " appétit moral », alors il manifesterait les traits caractéristiques de l'appétit : une attention accrue à des situations qui pourraient impliquer des considérations morales, ou aux dimensions morales d'une situation ; un sentiment de frustration à l'égard des circonstances si vous êtes 
empêché de tenir compte de ces considérations morales ; une colère envers vous-mêmes si vous n'arrivez pas à en tenir compte lorsque les circonstances le permettent ; une satisfaction particulière qui est indépendante d'autres motifs lorsque vous y parvenez; et ainsi de suite.

Remarquez que cet "appétit moral » ne peut entrer en jeu que chez un individu qui peut imaginer une situation (même de façon plutôt implicite) d'un point de vue moral, et qui possède donc des concepts moraux. C'est un appétit non pas pour des actes spécifiques, comme celui de porter secours, mais pour des actes par le biais d'une conception morale de ces actes, ou de soi-même. Ce ne serait donc pas simplement un appétit, mais un désir moral, entraînant avec lui l'ensemble habituel d'appétitions — la satisfaction liée au fait d'agir, la frustration liée au fait de ne pas réussir à agir — uniquement suite à et en réponse à l'évaluation morale de l'acte (encore une fois, il peut s'agir d'une évaluation très implicite). Il s'agit, en terminologie kantienne, d'un « intérêt de la raison ", parce que l'appétit s'est engagé vers l'action suite à ce que l'intellect ait pris conscience de son caractère moral.

Il écrit, dans une section de la Métaphysique des Mours intitulée "Prénotions esthétiques qualifiant la réceptivité de l'esprit aux concepts du devoir en général » :

Le sentiment moral. Celui-ci est la réceptivité au plaisir ou à la peine provenant uniquement de la conscience de l'accord ou du conflit entre notre action et la loi du devoir. Or, toute détermination de l'arbitre va de la représentation de l'action possible, à travers le sentiment de plaisir ou de déplaisir qu'on ressent à prendre un intérêt à cette action ou à son effet, jusqu'à l'acte; et là l'état esthétique, (l'affection du sens interne) est alors ou bien un sentiment pathologique ou bien un sentiment moral. Le premier est le sentiment qui précède la représentation de la loi, le second ce qui ne peut s'ensuivre que de celle-là. ${ }^{24}$

Kant situe le sentiment moral dans la faculté de désirer. Et la conscience active du devoir est impossible sans lui.

Or, ce ne peut être un devoir que d'avoir un sentiment moral ou d'en acquérir un, car toute conscience de l'obligation pose ce sentiment à son fondement ... Le fait est au contraire que tout homme (en tant qu'être moral) a en lui-même originairement ce sentiment, donc l'obligation peut uniquement consister à le cultiver et à le fortifier en allant jusqu'à admirer son origine insondable : c'est ce qui arrive lorsque l'on montre comment, séparé de tout attrait pathologique et pris dans sa pureté, c'est par la simple représentation de la raison qu'il est stimulé au plus haut point. ${ }^{25}$

J'ai choisi un exemple d'aide portée à un étranger impliquant très peu d'attrait préalable qui soit dû à des « intérêts de penchant ». Virtuellement

24. Ibid., vi, 399.

25. Ibid., vi, 399-400. 


\section{Philosophiques / Printemps 2001}

tous les penchants préalables pointent dans l'autre direction. Pourtant, il semble possible que vous puissiez néanmoins porter de l'aide dans un tel cas, tout comme il est intelligible qu'on puisse se gaver de nourriture sous les ordres du médecin, même lorsque la faim et le bon goût ne sont pas présents. Dans un tel cas, aucun appétit préalable ayant la forme d'une attente d'un « plaisir gustatif de l'objet » n'est présent — on mettrait avec plaisir la nourriture de côté si le médecin n'avait pas prescrit une telle ordonnance. Pourtant on voit aussi qu'un type d'appétit est également présent - vous serez satisfaits de vous-mêmes si vous voyez la nourriture s'achever, et vous ressentirez une frustration envers vous-mêmes si vous êtes incapable de la terminer. Votre désir de suivre l'ordonnance du médecin permet à un méchanisme appétitif proximal d'être déployé vis-à-vis d'une activité autrement peu alléchante, par le biais d'une idée de cette activité. Ainsi donc, on agit à la fois sur la base d'un désir (ce qui rend l'action explicable d'un point de vue psychologique) et à l'encontre d'un penchant préalable (retirez le jugement que la nourriture est requise pour la guérison et il ne resterait que de l'aversion pour l'action).

Dans le cas moral, Kant n'hésite pas à appeler le sentiment moral une "praedisposition" sous la forme d'une "propensio intellectualis ", ou un penchant " indépendant des sens ${ }^{26}$, ni de le situer dans la faculté de désirer. La "pureté » de la conviction que l'acte répond à notre sentiment moral c'est-à-dire l'assurance qu'on agit en fonction d'une idée morale plutôt que d'être uniquement guidé, en bout de ligne, par un "penchant du sentiment »- est fournie par notre emploi du test de l'impératif catégorique. Selon Kant, ce test me permet de déterminer ce qui, dans une situation donnée, est exigé d'un point de vue moral, indépendamment des "intérêts du sentiment » :

Sans expérience quant au cours du monde, incapable de parer à tous les événements qui s’y produisent, il suffit que je demande : peux-tu vouloir aussi que ta maxime devienne une loi universelle ?... Il serait ici aisé de montrer comment, ce compas à la main, [la raison humaine] a dans tous les cas qui surviennent la pleine compétence qu'il faut pour distinguer ce qui est bien, ce qui est mal, ce qui est conforme ou contraire au devoir ... ${ }^{27}$

Si Kant a raison, j’emploie le « test objectif » de l'impératif catégorique en l'appliquant aux "maximes subjectives »de mes actions, les évaluant ainsi à la lumière d'une idée spécifiquement morale. Cet exemple de délibération pratique a donc effectivement une motivation comme " prémisse ", et ne fournit pas une motivation, mais la " délibère " plutôt, l'évaluant à l'aide du test de l'impératif catégorique, et m'assurant qu'il s'y conforme avant d'en arriver à la conclusion pratique consistant à agir selon la maxime sub-

26. cf. Ibid., vi, 213.

27. Kant, Fondements de la Métaphysique des moeurs, 403-404. 
jective. Nous sommes enclins à mettre en pratique ce test de par notre "sentiment moral » préexistant, et, parce qu'il s'agit d'un désir véritable, nous éprouverons de la satisfaction envers nous-mêmes si, lorsque nous traduisons notre motivation en une action, nous employons avec succès le test catégorique - et nous éprouverons de la frustration ou de la colère envers nous-mêmes si nous n'y parvenons pas. Un appétit pour les actions accomplies par le biais d'un concept moral donne donc aux idées morales un rôle dans la régularisation du débit de la motivation sans qu'elles aient pour autant à générer de la motivation. Comme Kant l'exprime dans les Fondements, la nature n'a pas fait de la raison délibérative la source de la volonté ; elle a plutôt " délégué la raison au gouvernement de notre volonté " ${ }^{28}$. On retrouve ainsi au sein de notre faculté de désirer une " prédisposition (praedispositio) à être affectés par des concepts de devoir » qui se trouve " du côté du sentiment » et qui fait partie de notre don naturel en tant qu'êtres capables de raison pratique ${ }^{29}$. Comme le dirait peut-être Aristote, Kant nous a donné une façon de voir comment, étant donné que :

le choix est un désir délibératif, il faut par là même qu'à la fois la règle soit vraie et le désir droit, si le choix est bon, et qu'il y ait identité entre ce que la règle affirme et ce que le désir poursuit. Cette vérité et cette pensée dont nous parlons ici sont de l'ordre pratique... pour la partie de l'intellect pratique, son bon état consiste dans la vérité correspondant au désir, au désir correct. ${ }^{30}$

\section{Le désir est une forme d'appétit par l'entremise d'une idée, il n'est pas en-lui-même une idée ou un jugement}

Si le désir moral doit être distingué du penchant, il doit aussi l'être de ce que d'autres éthiciens ont appelé un "sens moral ». Kant rejette définitivement l'identification du désir au "sens moral » :

Il est impropre de désigner ce sentiment sous le nom de sens moral, en effet on entend généralement par le terme sens une faculté théorique de perception rapportée à un objet, tandis que le sentiment moral (comme le plaisir ou la peine en général) est quelque chose de purement subjectif qui ne délivre aucune connaissance. ${ }^{31}$

En effet, puisqu'il est un appétit pour les actes par l'entremise d'une représentation, le sentiment moral est une disposition à se soumettre à une cognition, mais ne constitue pas en elle-même une cognition. C'est, en partie, pourquoi aucun argument ou raisonnement théorique ne pourrait nous conduire à cette " prédisposition naturelle, » cette dernière fut-elle absente.

28. Ibid., 395.

29. Kant, Métaphysique des mours, 399.

30. Aristote, Éthique à Nicomaque, 1139a21-28.

31. Kant, Métaphysique des mours, vi, 400. 


\section{Philosophiques / Printemps 2001}

Il n'est pas d'homme dépourvu de tout sentiment moral, car si quelqu'un était entièrement dépourvu de réceptivité à cette sensation il serait, moralement parlant, mort, et si (pour parler le langage de la médecine) la force vitale morale ne pouvait plus exercer aucun attrait sur ce sentiment, l'humanité (comme sous l'effet de lois chimiques) se dissoudrait en la pure animalité et serait irréparablement confondue dans la masse des autres êtres naturels. ${ }^{32}$

Ainsi, ce sentiment ne juge pas les actions et ne peut être lui-même le produit d'un jugement ; il est plutôt un état constituant un appétit qui donne la force de motivation pour les actions, une fois qu'elles ont été jugées. Parce que son action ne dépend pas de quelque plaisir vécu ou envisagé qui soit occasionné par l'action en elle-même, mais d'un "intérêt de la raison » mis en marche suite à une évaluation de l'admissibilité morale de la maxime sur la base de laquelle nous agissons, le "fondement déterminant » de ce désir, et même "ce qui lui plaît ", dépend essentiellement de l'idée que se fait le sujet de l'action, et « se trouve dans la raison du sujet » ${ }^{33}$. Le plaisir que produit l'action issue de ce désir est conséquemment un " plaisir intellectuel ». Rappelons que :

[Si un plaisir précède nécessairement un désir comme cause], le plaisir pratique devient dans ce cas un intérêt du penchant; en revanche, lorsque le plaisir ne peut que suivre une détermination antérieure de la faculté de désirer, il devra être nommé plaisir intellectuel, et l'intérêt pris à l'objet, intérêt de la raison ... ${ }^{34}$

Voici donc l'exemple promis d'un désir qui constitue un véritable appétit, mais ne constitue pas un "penchant préalable ». Grâce à l'opération du raisonnement dans la mise en pratique de l'impératif catégorique, en conjonction avec un appétit pour l'action accomplie par l'entremise d'idées morales, l'action faite par devoir est possible, et est distincte de l'action qui s'accorde au devoir mais est accomplie en vertu d'un penchant préalable.

Suivant ces impératifs catégoriques, certaines actions sont licites ou illicites c'està-dire moralement possibles ou impossibles, et quelques-unes d'entre elles ou leur contraire sont moralement nécessaires, c'est-à-dire obligatoires. Intervient donc alors pour de semblables actions le concept d'un devoir dont l'exécution ou la transgression sont sans doute liées de surcroît à un plaisir ou un déplaisir d'un genre particulier (le genre du sentiment moral), lesquels plaisir ou déplaisir nous ne prenons toutefois aucunement en considération dans les lois pratiques de la raison [parce qu'ils ne concernent pas le principe des lois pratiques mais seulement l'effet subjectif que celles-ci exercent dans l'esprit lors de la détermination de notre arbitre et (sans rien leur ajouter ni leur ôter objectivement, c'est-à-dire à en juger en se plaçant du côté de la raison, de leur validité ni de leur influence) qu'ils peuvent varier selon la différence des sujets] $]^{35}$.

32. Ibid., vi, 400.

33. Ibid., vi, 213.

34. Ibid., vi, 212.

35. Ibid., vi , 221. Le texte entre crochets est tiré de l'original. 
Le fondement catégorique des lois pratiques est indifférent à nos sentiments personnels, moraux ou autres. Par conséquent, il offre un fondement objectif au devoir. Mais nous avons un intérêt pour des lois qui possèdent un tel caractère catégorique à cause d'un sentiment personnel, d'un sentiment moral, qui offre un fondement subjectif à la conduite morale. Selon Kant, la gouvernance normative prévaut lorsque ces deux éléments sont combinés, aucun des deux ne suffisant sans l'autre. Un sentiment moral dépourvu d'un test objectif pour le " réglementer » serait un genre de vœux consciencieux qui ne pourrait avoir comme résultat qu'une action satisfaisant nos prédilections morales personnelles - comme quelqu'un qui chercherait à être prudent mais ne pouvait juger l'intérêt à long terme de plusieurs lignes de conduite autrement qu'en considérant "l'effet qu'elles me font ». Un test objectif sans un sentiment moral qui puisse motiver de façon interne son application pratique pourrait, au plus, être un ordre qu'on reçoit, renforcé par une quelconque source d'incitation externe.

Plusieurs éthiciens ne seraient pas d'accord avec la conception kantienne de la nature du test objectif auquel doit est soumise l'action morale accomplie par devoir. Les utilitaristes, par exemple, soutiendraient qu'une évaluation impartiale de la valeur anticipée est un meilleur compas pour déterminer si nos buts personnels sont compatibles avec le fait de nous percevoir comme co-habitants d'un Royaume des fins. Et les contractariens contemporains proposent leurs propres standards. Mais les kantiens, utilitaristes et contractariens peuvent tous accepter qu'aucun standard objectif de ce type n'est " auto-motivant » - il offre un fondement objectif, et non subjectif, au devoir. Il n'y a aucune honte, aucune offense faite à la Raison en situant (comme le fait Kant) le fondement subjectif du devoir moral dans la faculté de désirer, en autant que le désir est (comme Kant le dit) un appétit pour une ligne de conduite par le biais d'une idée de sa conformité à un principe qui permet de déterminer de façon objective si celle-ci s'accorde avec notre citoyenneté dans le Royaume des Fins.

\section{Conclusion}

... le principe de l'action (humaine), c'est la décision ; (or) la décision, c'est une intelligence appétitive ou un appétit intellectif. ${ }^{36}$

Nous avons emprunté à Aristote l'idée que pour être pratique, la délibération doit avoir comme conclusion une action et doit par conséquent inclure ou présupposer la présence de tous les éléments de l'action, incluant le désir. L'action gouvernée par la raison pratique peut être conçue comme une sorte de "désir délibéré ", "intelligence appétitive », ou " appétit intellectif ». Mais comment le désir est-il délibéré ? Et comment est-ce qu'une action 


\section{Philosophiques / Printemps 2001}

véritablement accomplie par devoir peut-elle être basée sur un désir ? Nous avons considéré la réponse de Kant à de telles questions, lui empruntant la formulation explicite de l'idée selon laquelle le désir est un appétit dirigé vers un objet par le biais d'une représentation qui peut être obtenue via la pensée et le raisonnement. La gouvernance normative, pour Kant, est la gouvernance par des intérêts de la raison et a donc trait, comme Aristote le croyait, au fait d'exercer, de développer, de conduire à maturité, de renforcer, et d'ajuster la faculté de désirer. Notre vie pratique peut effectivement être de l'ordre du désir bien délibéré puisqu'elle est liée à la raison, celle-ci étant comprise comme la capacité de livrer des idées par le biais d'inférences.

Où se situe le « devrait» ( «ought») dans cette vision ? Considérez, d'abord, l'insistance de l'appétit. Même si nous ne prêtons pas attention à l'appétit, même s'il nous conduit à l'exténuement et à bien des difficultés, l'appétit exercera son influence sur notre conduite. La faim d'un animal peut le mener à quitter un lieu de cachette sécuritaire et à faire face au danger ; l'appétit que ressent une athlète pour la victoire peut la mener à s'entraîner pendant d'innombrables heures dans les circonstances les plus exigeantes, et à ressentir énormément de colère envers elle-même si elle ne le fait pas.

L'appétit est effectivement un type d'urgence subjective ou de nécessité qui entraînera des coûts pour un individu s'il ne lui porte pas une attention appropriée. Mais les êtres humains à qui ferait défaut un sentiment moral ne ressentiraient aucune urgence ou de " devrait » sur la base de considérations morales en elles-mêmes, comme c'est d'ailleurs le cas chez les bêtes et les petits enfants, à qui il manque une capacité à former des idées morales et à s'en servir dans la délibération.

L'archétype d'un " désir délibéré » ou d'un " appétit intellectif » est la gouvernance délibérative, au sein de laquelle un processus de raisonnement explicite donne lieu à une reconnaissance d'une condition objective qui active l'appétit en tant que tel, et en vient ainsi à posséder un degré d'urgence subjective. Mais ce n'est pas toute gouvernance normative ou rationnelle qui est ou pourrait être délibérative - certaines normes et certaines raisons, de par leur nature, exigent une réponse non-délibérée et, en général, une régression à l'infini serait initiée si la réponse appropriée à la force de la totalité des normes et raisons (y inclues celles de la délibération) devait être délibérative. Ce qui importe ce n'est pas tant que la délibération ait lieu, mais que soit identifiable une motivation s'accordant à une idée, à une image ou à un concept particulier, par le biais duquel la ligne de conduite incite notre engagement subjectif. Par exemple, les caractéristiques morales servant de raison d'agir, doivent expliquer, en partie, l'avènement d'un sentiment qui constitue un "sentiment moral ", et qui à son tour fonctionne comme une force motivationnelle bien accordée. La bonne volonté doit donc être trouvée dans la «faculté de désirer » plutôt que dans le domaine des processus de raisonnement pur, même si cette volonté réagit, de par sa nature, à certaines connaissances des circonstances nécessitant une action. 
Grâce au sentiment moral et à la conscience rationelle, nous pouvons ressentir l'urgence subjective d'un « devrait » objectif malgré la présence d'un penchant. Parce qu'il s'agit d'un véritable appétit, il peut susciter l'action nonobstant la difficulté ou le danger, donner lieu à une satisfaction motivante (un "plaisir pratique ») en dépit de l'effort et de la souffrance, et nous punir avec de la colère envers nous-mêmes si nous échouons. Parce qu'il est sensible aux considérations morales, parce qu'il est un appétit "par l'entremise de concepts ", il peut réagir à la force particulière des circonstances morales et à ce qu'elles requièrent (un "intérêt de la raison »). Évidemment, il se peut que notre perception des circonstances soit erronée, que nous manifestions de la faiblesse au niveau de notre appétit moral, ou encore que nous soyions distraits par d'autres idées et images. Il est donc fondamental à l'enseignement moral, pour Kant comme pour Aristote, que nous encouragions et développions le sentiment moral, promouvant ainsi au sein des agents un type d'habileté et de force dans le désir qui ne diffère pas en nature d'une habileté à être, par exemple, prudent ou brave. 